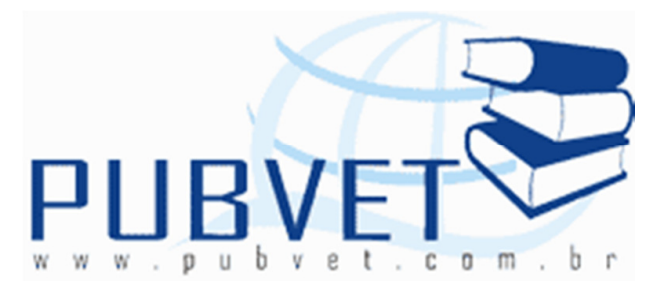

PUBVET, Publicações em Medicina Veterinária e Zootecnia.

\title{
Receptores de estrógeno e seus ligantes: uma breve revisão
}

Paulo Alex Bezerra Sales ${ }^{1}$, Andréia da Silva Costa ${ }^{1}$, Jamylla Mirck Guerra de Oliveira ${ }^{1}$, Kleverton Ribeiro da Silva ${ }^{2}$, Amilton Paulo Raposo Costa ${ }^{3}$

1 Doutorando(a) em Ciência Animal - UFPI

2 Doutorando em Patologia Experimental- FIOCRUZ-UFBA

3 Professor Doutor - UFPI

\section{Resumo}

O uso de estrógenos na terapia de reposição hormonal é muito amplo, visando reparar os danos fisiológicos causados pela deficiência desse hormônio em certos períodos da vida como, por exemplo, na menopausa. Vários são os tipos de receptores estrogênicos, e há uma infinidade de possíveis ligantes. Estes agem de forma diferente, na maioria das vezes modulando os receptores de estrógeno, suprindo ou inibindo as ações desse hormônio. Assim, torna-se importante o conhecimento do modo de ação dessas substâncias e como elas podem regular as necessidades fisiológicas do organismo como coadjuvantes do tratamento de diversas patologias endócrinas.

Palavras-chave: estrógenos; serms; fitoestrógenos; receptores. 
SALES, P.A.B. et al. Receptores de estrógeno e seus ligantes: uma breve revisão. PUBVET, Londrina, V. 8, N. 15, Ed. 264, Art. 1755, Agosto, 2014.

\title{
Estrogen receptors and their ligands: a brief review
}

\begin{abstract}
The use of estrogen in hormone replacement therapy is very wide, being necessary to repair the damage caused by physiological deficiency of this hormone in certain periods of life, such as menopause. There are several types of estrogen receptors, and there are plenty of potential ligands. These act differently, mostly by modulating the action of estrogens receptors, supplying or inhibiting the actions of this hormone. Thus, it becomes important to know the mode of action of these substances and how they may regulate the physiological needs of the body as adjuvant treatment of various endocrine pathologies.
\end{abstract}

Keywords: estrogens; serms; phytoestrogens; receptors.

\section{1- INTRODUÇÃO}

Os estrógenos são hormônios que produzem numerosas ações fisiológicas, incluindo efeitos no desenvolvimento, ações neuroendócrinas envolvidas no controle da ovulação, preparo cíclico do trato reprodutor para fertilização e implantação do óvulo bem como ações no metabolismo de minerais, carboidratos, proteínas e lipídios. O uso terapêutico dos estrógenos é disseminado, sendo comumente utilizado na reposição hormonal após a menopausa e na anticoncepção [1].

A maioria, se não todos, dos efeitos conhecidos dos estrógenos são mediados por fatores de transcrição ativados por ligantes, chamados de receptores de estrógeno (REs) [2]. Esses receptores são pertencentes à superfamília de receptores nucleares e subdividem-se em vários domínios funcionais. Os mais conhecidos e estudados subtipos de receptores estrogênicos são os receptores a (REa), o qual foi clonado em 1986, e $\beta$ (RE $\beta$ ) clonado em ratos [3] e em humanos [4]. 
SALES, P.A.B. et al. Receptores de estrógeno e seus ligantes: uma breve revisão. PUBVET, Londrina, V. 8, N. 15, Ed. 264, Art. 1755, Agosto, 2014.

A comunicação celular é algo fundamental para a homeostase do organismo vivo, pois sabemos das interligações entre os diversos sistemas, onde observamos dependências cruciais entre os mesmos. Essas comunicações ocorrem através de sinais químicos, sejam eles de origem endócrinos, parácrinas ou autócrinas. Várias doenças acometidas de forma universal estão ligadas a deficiência nessas comunicações, podendo o problema ser gerado na origem da sinalização que se dá através de substâncias específicas como é o caso dos hormônios, ou no receptor específico que recebe essa sinalização. 0 estudo sobre a presença de ligantes específicos para receptores específicos se torna uma ferramenta fundamental para o conhecimento de inúmeras patologias e consequentemente a descoberta do controle e até mesmo a cura dessas doenças onde aumentaria significativamente a qualidade de vida.

Estudos promissores estão sendo realizados a nível mundial sobre receptores de estrógeno que assumem papéis importantes nessa qualidade de vida, sendo na melhora clínica do quadro do paciente ou na profilaxia de diversas doenças, dentre elas o câncer. Dessa forma, o foco dessa revisão foi elucidar a importância dos principais ligantes dos receptores estrogênicos, tendo em vista seus papéis em diversos distúrbios endócrinos.

\section{2- REVISÃO DE LITERATURA}

\section{1- RECEPTORES DE ESTRÓGENO}

A partir dos anos 70 Jensen e Desombre, 1997 caracterizaram os receptores de estrógenos como pertencentes à família de receptores nucleares [5]. Até meados dos anos 90 acreditava-se que só existia um tipo de receptor de estrógeno, o Receptor de Estrógeno a (REa), mas outro tipo de receptor foi encontrado, o Receptor de Estrógeno $\beta$ (RE $\beta$ ), sendo evidenciados e estudados em humanos e ratos $[3 ; 6]$.

Atualmente o estudo sobre receptores de estrogênios está em contínua ascensão, onde já se sabe que tanto o REa e RE $\beta$ possuem distinção celular e 
SALES, P.A.B. et al. Receptores de estrógeno e seus ligantes: uma breve revisão. PUBVET, Londrina, V. 8, N. 15, Ed. 264, Art. 1755, Agosto, 2014.

diferenciação de distribuição tecidual [7]. Os domínios de ligação ao DNA do REa e RE $\beta$ apresentam alto grau de homologia (97\%), mas o domínio de ligação ao ligante apresenta apenas $57 \%$ de homologia, entendendo que essas diferenças possibilitem desenvolver ligantes REa e RE $\beta$ específicos [8].

A localização desses receptores já tem sido constatada e estudada em diversos órgãos. Sabemos que alguns tecidos possuem receptor tipo $\beta$, mas não possuem a sugerindo que o estrogênio possua ação em alguns tecidos por ser mediados por ativação de $\beta$ em vez de a e vice - versa, e ainda afirmam que embora a distribuição de RE $\beta$ pareça estar intimamente relacionada a expressão de REa a expressão de RE $\beta$ não parece estar ligado a expressão de REa [6].

Os RE são amplamente distribuídos no organismo. Em tecidos do córtex cerebral, cerebelo, miocárdio, pulmão, esôfago, pituitária, tireoide, próstata, testículo, ovário, vagina, útero e mama já foram constatados a presença de RE $\beta$ [6]. Tecidos como útero, mama e pituitária são alvos já bem conhecidos de REa, mas sabemos que eles estão presentes em diversos outros tecidos como ósseo, cardiovascular e sistema nervoso central [9;10]. Já em hepatócitos e hipocampo somente foram encontrados REa [6].

Comparando estudos realizados em tecidos humanos [6] e em tecidos de ratos [3] observou-se que a presença de RE $\beta$ no testículo e próstata foi observada de forma semelhante, e que a falta de REa na próstata com uma presença significativa de $R E \beta$ nos levam a concluir que o tratamento clinico do câncer de próstata, por exemplo, só pode ser mediada através de um RE $\beta$ dependente [11].

A presença de RE $\beta$ no útero era algo controverso onde Taylor e Al-Azzawi [6] afirmam sua presença, Couse [12] fala de sua ausência e Couse [13] diz que o mesmo possui uma fraca expressão. Nesse mesmo estudo feito por Taylor e Al-Azzawi [6] dados sugerem que alguns tecidos somente expressam RE $\beta$ como, por exemplo, a próstata, dando margem para uma investigação 
SALES, P.A.B. et al. Receptores de estrógeno e seus ligantes: uma breve revisão. PUBVET, Londrina, V. 8, N. 15, Ed. 264, Art. 1755, Agosto, 2014.

mais minuciosa quando o assunto abordado é câncer de próstata. Em outros estudos observou-se a ausência de REa na próstata em virtude da não reação do anticorpo a, enquanto no útero a sua reação foi constatada [14]. Verificouse ainda a ausência de RE $\beta$ no corpo lúteo, contradizendo os estudos de Saunders et al. [15] em que a presença de RE $\beta$ no corpo lúteo foi confirmada.

\section{2- ESTRÓGENO LIGANTE NATURAL}

A relevância do hormônio estrogênio é algo não discutível, pois sabemos de sua atuação em diversos processos fisiológicos, como no crescimento, diferenciação e na função de tecidos do sistema reprodutivo (útero, vagina, glândulas mamárias, testículos e próstata) $[16 ; 17]$. Atua ainda mantendo a densidade óssea, agindo como cardioprotetor e possuindo efeito sobre os lipídeos do sangue, bem como regula o comportamento reprodutivo e a produção de gonadotrofinas $[18 ; 19 ; 20]$.

O aumento ou um déficit desse hormônio leva a uma série de consequências aos seres humanos e animais, o que faz necessários estudos mais aprofundados sobre o mesmo.

Os estrógenos participam dos processos de crescimento, diferenciação, maturação e funcionamento dos neurônios [21]. Um dos sistemas neurais em que o estrógeno parece atuar como modulador é o sistema da acetilcolina, fonte primária de inervações no hipocampo, que está envolvido nos processos gerais de atenção, aprendizado e formação de memória [22].

Sabe-se que o estrógeno está envolvido tanto na prevenção como na indução do câncer prostático. Uma excessiva exposição ao estrógeno durante etapas críticas do desenvolvimento leva a neoplasia prostática [23], já quando se faz uso de tratamentos ricos em fitoestrógenos em ratos adultos, constatouse que há diminuição significativa do peso da próstata [24] levando a crer que ele possa ter um efeito protetor sobre tal orgão no que diz repeito ao câncer de próstata. 
SALES, P.A.B. et al. Receptores de estrógeno e seus ligantes: uma breve revisão. PUBVET, Londrina, V. 8, N. 15, Ed. 264, Art. 1755, Agosto, 2014.

\section{3- MODULADORES SELETIVOS DE RECEPTORES DE ESTRÓGENOS}

Atualmente para suprir ou inibir a ação hormonal de estrógenos faz-se uso de compostos sintéticos denominados SERMS (Moduladores seletivos de receptores de estrógenos) que podem agir de forma agonista ou antagonista, e com respostas estrogênicas ou antiestrogênicas, pois alguns atuam antagonizando os efeitos estimulatórios do estrógeno [26].

Esses moduladores são classificados em antiestrógenos puro (ICL164384), de primeira geração (tamoxifeno) e segunda geração (raloxifeno). Eles agem de formas diferentes dependendo do tecido alvo e em virtude de inúmeros mecanismos tal como por exemplo a presença de receptores diferentes, levando também em consideração a própria distribuição desses receptores nos tecidos. Exemplo clássico tem o raloxifeno onde o mesmo atua como um legítimo agonista no tecido ósseo e antagonista no tecido mamário. É importante frisar que os mecanismos pelos quais agem de forma seletiva ainda não são completamente conhecidos [20].

Um SERM bastante utilizado atualmente é o Raloxifeno, derivado benzotiofênico, que possui afinidade pelo receptor de estrógeno semelhante ao $17 \beta$ estradiol. Alguns estudos falam da sua capacidade de estimular as vias estrogênicas através de receptor $\beta$, mas não são capazes via receptor $a$, dando margem para levarmos em consideração a sua ação diferencial nos tecidos $[26 ; 27]$.

A habilidade dos SERMS suprimirem a carcinogênese de vários cânceres já é conhecida, dentre eles o de próstata, onde esse tipo de câncer tem se tornado bastante comum, aonde sua incidência vem aumentando em muitos países. Estudos com Toremifene e posteriormente com Raloxifeno, revelaram uma regressão prostática e das vesículas seminais em ratos Sprague-Dawlley [28]. 
SALES, P.A.B. et al. Receptores de estrógeno e seus ligantes: uma breve revisão. PUBVET, Londrina, V. 8, N. 15, Ed. 264, Art. 1755, Agosto, 2014.

Um dos grandes avanços para o uso do raloxifeno é no que tange a atividade inibitória estrogênica no tecido mamário [20]. Cummings [29] verificou em seu estudo que o raloxifeno diminuiu $76 \%$ a incidência de novos casos de câncer invasivo.

O efeito antagonista do raloxifeno sobre o endométrio teve resultados bastante significantes, onde a hipertrofia endometrial induzida pelo estradiol e o crescimento de carcinoma endometrial provocado pelo tamoxifeno são inibidos quando se administrava raloxifeno [27;30].

Por se tratar de um medicamento já utilizado em várias enfermidades, mas ainda em constantes estudos, a continuação de trabalhos com o intuito de evidenciar novos efeitos e para comprovar ou não o que diz a literatura, vê-se a real necessidade de seu estudo mais aprofundado.

\section{4- FITOESTRÓGENOS}

Outra forma de suprir ou inibir a ação hormonal de estrógenos no organismo é fazer o uso de substâncias denominadas de fitoestrógenos, que possuem atividade biológica semelhante ao estrógeno. Estudiosos já evidenciaram que são compostos fenólicos heterocíclicos com similaridades estruturais com estrógenos sintéticos e naturais [31].

Essa atividade estrogênica das plantas foi primeiramente demonstrada em 1926, e em meados da década de 70 já se tinha demonstrado que centenas de plantas exibiam atividade estrogênica. Os fitoestrógenos assumiriam importância biológica e econômica nos anos 40, com a diminuição da fertilidade induzida em ovelhas pela ingestão de trevos de pastagens, na Austrália, na chamada "Doença do Trevo" [32].

Estima-se que aproximadamente 5 a $15 \%$ de um total de 250.000 espécies de plantas superiores tenham sido sistematicamente investigadas para a presença de compostos bioativos [33] o que demonstra a importância de ser dada continuidade a esses estudos [34]. SIMONS; GRINWICH [35] 
SALES, P.A.B. et al. Receptores de estrógeno e seus ligantes: uma breve revisão. PUBVET, Londrina, V. 8, N. 15, Ed. 264, Art. 1755, Agosto, 2014.

realizaram estudos para verificar a ocorrência de esteroides em plantas utilizando a técnica de radioimunoensaio, sendo estudas 128 plantas de mais de 50 famílias, ocorrendo resultado positivo para 109 dessas. Androsterona e progesterona foram encontrados em mais de $80 \%$ das espécies estudadas, testosterona e diidrotestosterona em $70 \%$ e estrogênios como estrona e $17 \beta$ estradiol em $50 \%$ das espécies.

As três principais classes de fitoestrogênios são: Isoflavona, sendo os de maior relevância o genisteine e a daidzein; Coumestano, tendo o coumestrol como principal representante e por fim os Lignanos representados prinicipalmente pelo esterodiol e a enterolactona $[31 ; 36]$. Existe ainda uma quarta classe de fitoestrógenos que se refere aos micoestrógenos (lactonas do ácido resorcílico), representados pela zearalenona e zearalenol [36].

O mecanismo pelo qual eles atuam no organismo já é conhecido. Na ingestão de fitoestrógenos eles sofrem conversões enzimáticas e metabólicas no trato digestivo resultando em fenóis heterocíclicos com grande similaridade com estruturas dos estrogênios sendo absorvidos na circulação enterohepática podendo ser excretado na bile, desconjugados pela flora intestinal, reabsorvidos, reconjugados pelo fígado e excretados pela urina [32].

Ao levar em consideração a potência dos fitoestrógenos, temos que fazer algumas colocações. As ações estrogênicas dos fitoestrogênios são extremamente fracas comparados ao estrógeno natural [37] e eles atuam fisiologicamente muito mais como anti-estrogênios do que como estrogênios. Os efeitos exercidos pelos fitoestrógenos parecem ser resultados de sua ligação ao receptor beta $[39 ; 40]$. Exemplo disso tem a genisteína que se liga 5 a 20 vezes com mais afinidade pelo receptor estrogênico ER $\beta$ do que ao ERa [41]. A maior afinidade ao receptor ER $\beta$ sugere que as isoflavonas podem exercer efeitos seletivos (estrogênico e antiestrogênico), dependendo do tecido e da concentração de isoflavonas. Já o estradiol de mamíferos tem maior afinidade por receptores estrogênicos ERa, presentes no tecido mamário e 
SALES, P.A.B. et al. Receptores de estrógeno e seus ligantes: uma breve revisão. PUBVET, Londrina, V. 8, N. 15, Ed. 264, Art. 1755, Agosto, 2014.

uterino [38]. Essa diferença na afinidade pelos receptores pode explicar como os fitoestrógenos beneficiam alguns sistemas sem influenciar em outros [42].

Estudos recentes têm demonstrado os efeitos benéficos dos fitoestrógenos na prevenção de várias doenças crônicas como câncer de cólon, mama, próstata, doenças cardiovasculares e principalmente, os efeitos benéficos em pacientes na pós-menopausa, no sentido de reduzir os sintomas e prevenir as doenças decorrentes da síndrome de climatério como a osteoporose $[43 ; 44 ; 45 ; 46 ; 47 ; 48 ; 49 ; 50]$.

Muitos medicamentos utilizados pela indústria farmacêutica são de origem vegetal, chegando a $25 \%$ dos que são prescritos, equivalendo a 121 substâncias ativas usadas na terapêutica seja para o tratamento de doenças ou na prevenção [51].

Várias plantas consumidas por animais e seres humanos têm sido estudadas profundamente em virtude dos efeitos que as mesmas podem vir a ocasionar sobre o organismo, podendo ser maléfica ou benéfica. Podemos destacar como alvo de estudos: Soja (Glycine max), Trevo-dos-prados (Trifolium pratense), Black cohosh (Cimicífuga racemosa), Dong quai (Angelica sinensis), Alcaçuz (Glycyrrhiza glabra), Chaste tree berry (Vitex agnus-castus), Ginseng (Panax ginseng), Óleo de prímula (Oenethera biennis), Romã (Punica granatum L.), Amora (Morus nigra L), Ipoméia (Ipomoea cairica), Sacaca (Croton cajucara Benth), Melão-de-São-Caetano (Momordica charantia), Espinheira Santa (Maytenus ilicifolia Mart). Atualmente várias pesquisas tem sido desenvolvidas com ratos de laboratório (dentre outras espécies) demonstrando a importância dos hormônios esteróides, principalmente o estrógeno, para a manutenção e controle de diferentes funções fisiológicas, como metabolismo de lipídeos e diversas funções cerebrais, podendo assim dar respostas significativas aos homens e animais.

No Brasil, a utilização de plantas como meio curativo é uma atividade altamente difundida e popular, às vezes, empregadas de maneira errônea, 
SALES, P.A.B. et al. Receptores de estrógeno e seus ligantes: uma breve revisão. PUBVET, Londrina, V. 8, N. 15, Ed. 264, Art. 1755, Agosto, 2014.

afinal muitas plantas possuem princípios tóxicos e o seu uso indiscriminado pode causar sérios problemas, por isso se faz necessário o estudo dos reais efeitos das mesmas sobre os homens e animais.

Muitas plantas, no Estado do Piauí, são utilizadas popularmente com indicações específicas para alterações do ciclo menstrual, como contraceptivo e abortivo. Tais indicações nos levam à suspeita de atividade estrogênica ou embriotóxica nessas plantas. Sabendo-se que muitas delas são consumidas pelos animais domésticos e outras são utilizadas para tratamento de suas enfermidades, faz-se necessário a utilização de protocolos específicos para verificar tais efeitos, visto que tais efeitos podem levar a transtornos reprodutivos nos animais atuando em receptores específicos de estrógeno.

\section{3- CONCLUSÃO}

Os estrógenos desempenham importantes funções, tanto no sistema reprodutivo quanto nos demais tecidos, a exemplo do tecido ósseo. A diminuição da produção endógena desses hormônios deve ser acompanhada de reposição exógena, visto que sua ausência desencadeia uma série de patologias como desmineralização óssea, distúrbios cardiovasculares entre outros. Uma boa alternativa à reposição com a forma sintética do hormônio é o uso de fitoestrógenos, como as isoflavonas, que se ligam de forma seletiva aos receptores estrogênicos modulando seus efeitos, de modo semelhante aos moduladores seletivos de receptores de estrógeno (SERMS), diminuindo, de certa forma, os efeitos colaterais resultantes do tratamento com a forma sintética do estradiol.

\section{REFERÊNCIAS BIBLIOGRÁFICAS}

1. WILLIAMS, C. L.; STANGEL,G. M. Estrógenos e progestogênios. In: GOODMAN. GILMAN. As bases farmacológicas da terapêutica. 9. ed. Rio de Janeiro: Mc Graw Hill, 1996. cap. 57, p. 1045-67. 
2. ENMARK, E.; GUSTAFSSON, J.-AÊ. Oestrogen receptors - an overview. Journal of Internal Medicine, v.246, p.133 -138, 1999.

3. KUIPER, G.G et al. Cloning of a novel receptor expressed in rat prostate and ovary. Proc Natl Acad Sci., v. 93, p.5925-5930, 1996.

4. MOSSELMAN S, POLMAN J, DIJKEMA R. ERß: identification and characterization of a novel human estrogen receptor. FEBS Lett, v.392, p.49-53, 1996.

5. O'DONNELL, L. et al. Estrogen and spermatogenesis. Endocrine Reviews, v.22. p. 289-318, 2001.

6. TAYLOR, A.H.; AL-AZZAWI, F. Immunolocalisation of oestrogen receptor beta in human tissues. J Mol Endocrinol., v.24, p.145-155.

7. DIAZ, L.K.; SNEIGE, N. Estrogen receptor analysis for breast cancer: current issues and keys to increasing testing accuracy. Adv Anat Pathol., V.12, p.09-10,2005.

8. GUSTAFSSON, J.A. Estrogen receptor $b-a$ new dimension in estrogen mechanism of action. J Endocrinol., v.163, p.379-383, 1999.

9. ZHAO, L.; BRINTON, R.D. Structure-based virtual screening for plant-based ERbetaselective ligands as potential preventative therapy against age-related neurodegenerative diseases. J Med Chem., v.48, p.3463-3466, 2005.

10- COUSE, J.F.; KORACH, K. S. Estrogen receptor null mice: what have we learned and where will they lead us. Endocrinogy, v.20, p.358-417, 1999.

11- CARLSTROM, K. et al. Possible bone-preserving capacity of high-dose intramuscular depot estrogen as compared with orchidectomy in the treatment of patients with prostatic carcinoma. Prostate. v.31 p.193-197, 1997.

12- COUSE, J.F. et al. Quantitative analysis and distribution of transcripts enconding the estrogen receptor subtypes ERa and ER $\beta$ in tissues of the wild type and erko mouse. 79th Annual Meeting of the Endocrine Society.

13- COUSE, J.F. et al. Tissue distribution and quantitative analysis of estrogen receptoralpha (ER) and estrogen receptor-beta (EB) messenger ribonucleic acid in the wild-type and ER-knockout mouse. Endocrinology v.138, p.4613-4621,1997.

14- HIROI, H. DiVerential immunolocalization of estrogen receptor á and â in rat ovary and uterus.Journal of Molecular Endocrinology. v.22, p.37-44, 1999.

15- SAUNDERS, P.T.K. et al. Expression of oestrogen receptor beta (ERbeta) in multiple rat tissues visualised by immunohistochemistry. Journal of Endocrinology, v.154 p.13-16, 1997.

16- FRANZOTTI, E.M., Identificação de agonistas e antagonistas de receptores nucleares em extratos de plantas medicinais: Morus nigra L.,Plextranthus Ornatus Codd., Ipomoea Cairica(L) Sweet e Pouteria Torta (Mart.) Radlk .2006.108f.Tese (Doutorado em ciências da saúde) - Programa de pós - graduação em ciências da saúde,Universidade de Brasília, Brasília, 2006. 
17- KUIPER, G.G. et al.. Cloning of a novel estrogen receptor expressed in rat prostate and ovary. Proc Natl Acad Sci. v.93,p.5925-30,1996.

18- OSBORNE, C.K.; ZHAO, H.H.; FUQUA, S.A.W. Selective estrogen receptor modulators: structure, function, and clinical use. J Clin Oncol; v.18,p.3172-3186, 2000.

19- SCHMIDT, C. et al.Perfil lipoprotéico de cadelas submetidas à ovário-histerectomia com e sem reposição estrogênica. Arq. bras. med. vet. Zootec. v.56, p.449-456, 2004.

20- KAYATH, M. J. Raloxifeno e Osteoporose: Revisão de um novo modulador seletivo do receptor de estrógeno. Arq. Bras. Endocrinol. Metab.. v..43, p.433-440,1999.

21- BELCHER, S. M.; ZSARNOVSZKY, A. Estrogenic actions in the brain: Estrogen, Phytoestrogens, and rapid intracellular signaling mechanisms. JPET, v.299, p.408-414, 2001.

22- DANIEL, J. M. ; DOHANICH, G.P. Acetylcholine mediates the estrogen-induced increase in NMDA receptor binding in CA1 of the hippocampus and the associated improvement in working memory. J. Neurosci. v.21, p.6949-6956, 2001.

23- MESSINA, M. J. et al. Soy intake and cancer risk: a rev i ew of the in vitro and in vivo data. Nutr Cancer, v.21, p.113-131, 1994.

24- WEBER, K. S. et al. Dietary soy-phytoestrogens decrease testoterone levels and porstate weight without alterinh $\mathrm{LH}$, prostate 5a-reductase or testicular steroidogenic acute regulatory peptide levels in adult mat Sprague-Dawley rats. Journal of endocrinology.v.170, p.501-599, 2001.

25- BRYANT, H.U., et al. A pharmacological review of raloxifene. J. Bone Miner. Metab., v.14, p.1-9, 1995.

26- MCDONNELL DP, ET AL. Analysis of estrogen receptor function in vitro reveals three distinct classes of antiestrogens. Mol Endocrinol, v.9, p.659-969, 1995.

27- BRZOZOWSKI A.M. et al. Molecular basis of agonism and antagonism in the oestrogen receptor. Nature. v.389:p.753-758, 1997.

28- NEUBAUER, B.L. et al. Endocrine and antiprostatic effects of raloxifene (LY 156758) in the male rat. Prostate, v.23, p.246-262, 1993.

29- $\quad$ CUMMINGS, S.R. et al. The effect of raloxifene on risk of breast cancer in postmenopausal women - results from the more randomized trial. JAMA.p.281:2189, 1999.

30- GOTTARDIS M.M. et al. Effect of steroidal and non steroidal antiestrogens on the growth of a tamoxifen-stimulated human endometrial carcinoma (EnCa 101) in athymic mice.Cancer Res. v.50, p.3189-3192, 1990.

31- CHIECHI, L.M.Dietary phytoestrogens in the prevention of long-term ostmenopausal diseases. Int J Gynecol Obstet, v.67,p.39-40, 1999.

32- MURKIES A.L.; WILCOX G.; DAVIS S.R. Clinical review 92: Pytoestrogens. J Clin Endocrinol Metab., v. 83, p. 297-303, 1998.

33- CRAGG, G. M.; NEWMAN, D. J. Discovery and development of antineoplastic agents from natural sources. Cancer Invest, v.17, p.153-163, 1999. 
34- $\quad$ ALEXANDROVA, R. et al. "Phytoproducts and cancer." Experimental Pathology and Parasitology, v. 4, p. 15-26, 2000.

35- SIMONS, R.G.; GRINWICH D.L. Immunoreactive detection of four mammalian steroids in plants. Can J Bot, v.67, p.288-296,1989.

36- ADLERCREUTZ, $\mathrm{H}$. et al.Maternal and neonatal phytoestrogens in japonese women during birth. Am J Obstet Gynecol,v.180,p.737-743,1999.

37- MARKIEWICZ, L. et al. In vitro bioassays of non steroidal phytoestrogen. J. Steroid Biochem. Molec. Biol. V.45, P.399-405,1993

38- KUIPER, G.G.J.M. et al Interaction of estrogenic chemicals and phytoestrogens with estrogen receptor beta. Endocrinology. v.130, p.4252-4263, 1998.

39- PETERSEN, D.N. ET AL. Identification of estrogen receptor b2, a functional variant of estrogen receptor b expressed in normal rat tissues. Endocrinology, v.139, p.1082$1092,1998$.

40- $\quad$ CASSIDY, A. Potential tissue selectivity of dietary phytoestrogens and estrogens. Curr.Op. Lipidol, v.10, p. 32-47,1999.

41- KUIPER, G.G.J.M.; GUSTAFSSON, J.A. Ligand binding specificity and tissue distribution of rat estrogen receptor subtype. 79th Annual Meeting of the Endocrine Society, p.403-429. 1997.

42- DUNCAN, A.M. et al. Premenopausal equol excretors show plasma hormone profiles associated with lowered risk of breast cancer. Cancer Epidemiol Biomarkers Prev v.9, p.581-586, 2000.

43- $\quad$ CLARKSON, T.B. et al. The potential of soybean phytoestrogens for postmenopausal hormone replacement therapy. Proceedings of the Society for Experimental Biology and Medicine, v.217, p. 365-368, 1998.

44- $\quad$ MURKIES, A.L.et al. Dietary fluor supplementation decreses post-menopausal hot flusher: Effect of soy and wheat. Maturitas, v. 21, p.189-195,1994.

45- $\quad$ AGNUSDEI, D.; BUFALINO, L. Efficacy of ipriflavone is established osteoporosis and long-term safety. Calcified Tissue International, v. 61, p. $23-27,1997$.

46- $\quad$ ANDERSON, J.J.B.; GARNER, S.C. The effects of phytoestrogens on bone. Nutrition Research, v. 17, p.1617-1632,1997.

47- THAM, D.M.; GARDNER, C.D.; HASKELL, W.L. Potential health benefits of dietary phytoestrogens: a review of the clinical, epidemiological and mechanistic evidence. J. Clin. Endocrinol. Metab., v.83, p. 2223-2235,1998.

48- GENNARI, C. et al. Effect of chronic treatment with ipriflavone in postmenopausal women with low bone mass. Calcif. Tissue Int., p.19-22, 1997.

49- BARNES, S. Evolution of the health benefits of soy isoflavonas. J. Soc. For Exp. Biol. And Med., v.217, p. 386-392, 1998

50- DAVIS S.R. et al. Phytoestrogens in health and disease. Recent Prog Horm Res, v.54, p.185-210. 1999. 
51- HAMBURGER, M.; HOSTETTMANN K. Bioactivity in plants: between phytochemistry and medicine. Phytochemistry, v.30, p. 3864-3874,1991. 